\title{
The effect of CYP2C9 and VKORC1 genetic polymorphisms on warfarin dose requirements in a pediatric population
}

\author{
Birce Dilge Taşkın, Serdar Kula1, Mehmet Ali Ergün², Demet Altun4, Rana Olguntürk1, \\ Fatma Sedef Tunaoğlu ${ }^{1}$, Ayşe Deniz Oğuz ${ }^{1}$, Türkiz Gürsel ${ }^{3}$
}

Department of Pediatric Neurology, Ankara Children’s Hematology Oncology Training and Research Hospital, Ankara-Turkey Department of ${ }^{1}$ Pediatric Cardiology, ${ }^{2}$ Medical Genetics, ${ }^{3}$ Pediatric Hematology, Faculty of Medicine, Gazi University, Ankara-Turkey Department of ${ }^{4}$ Pediatrics, Etimesgut Air Force Hospital, Ankara-Turkey

\section{ABSTRACT}

Objective: The aim was to investigate the frequency of genetic polymorphisms of cytochrome P4502C9 (CYP2C9) and vitamin K epoxide reductase complex subunit1 (VKORC1) and determine the effect of these polymorphisms on warfarin dose requirements in pediatric patients.

Methods: Fifty-eight pediatric patients with cardiac disease, thrombophilia, or other conditions, taking a stable warfarin dose, aged 0.2-18 years, and with international normalized ratio (INR) between 2 and 3 and 149 healthy children as a control group were included in this prospective, observational study. Patients receiving drugs that interact with warfarin, having chronic liver or renal disease, obesity, or thyroid dysfunctions were excluded. Polymerase chain reaction (real time and restriction fragment length polymorphism) was used to analyze the CYP2C9*2, CYP2C9*3, and VKORC1 polymorphisms. The ideal warfarin dose was calculated according to the patient's age, height, and the presence of CYP2C9*2, CYP2C9*3, and VKORC1 genetic polymorphisms. The mean daily administered doses and ideal doses were compared. Analysis of variance, Student's t-test, logistic regression analysis, and Pearson's correlation analysis were used for statistical analyses.

Results: The frequency of the CYP2C9 and VKORC1 genetic polymorphisms was determined as CYP2C9*1/*1 (54.6\%), * $1 /{ }^{*} 2(16.4 \%),{ }^{*} 1 /{ }^{*} 3(24.2 \%)$, ${ }^{*} 2{ }^{*} 3(2.9 \%),{ }^{*} 3 /{ }^{*} 3(1.9 \%)$, wild-type VKORC1 $(26.6 \%)$, heterozygote alleles $(52.7 \%)$, and mutant alleles $(20.8 \%)$. Patients with allelic variants were found to require lower warfarin doses, and a $64.5 \%$ correlation was found between the calculated ideal doses and the administered warfarin doses. Conclusion: Considering CYP2C9 and VKORC1 genetic polymorphisms prior to commencing warfarin treatment will make it easier to reach target INRs and reduce the rate of complications. (Anatol J Cardiol 2016; 16:000-00)

Keywords: CYP2C9, genetic polymorphism, INR, pharmacogenetics, VKORC1, warfarin

\section{Introduction}

For decades, warfarin has been the most commonly prescribed anticoagulant for the prophylaxis and treatment of venous and arterial thromboembolic disorders. Despite the limitations of warfarin, including a narrow therapeutic range, broad variations in intra- and inter-individual drug requirements, and a relatively high incidence of bleeding complications, its use continues to rise (1-3). The main reasons for its worldwide use are as follows: the drug can be orally administered and its low cost (1-3). The treatment efficacy and safety of the use of warfarin are based on the prothrombin time, which is expressed as the international normalized ratio (INR).

The narrow therapeutic range of warfarin means that the effective dose differs among individuals. The latter also has impli- cations in follow-up therapy. Both non-genetic and genetic factors play a role in the metabolism of warfarin. The non-genetic factors involved in the metabolism of the drug are age, race, body mass index (BMI), gender, smoking, concomitant renal or hepatic disease, drug therapy, enteral feeding, impaired absorption or rapid elimination of vitamin $\mathrm{K}$, and dietary vitamin $\mathrm{K}$ intake (4-6). Two genes, the cytochrome P450 complex subunit 2C9 (CYP2C9) and vitamin $\mathrm{K}$ epoxide reductase complex subunit 1 (VKORC1), are mainly responsible for the metabolism of warfarin.

CYP2C9, which plays a role in the metabolism of many drugs other than warfarin, is a liver enzyme. The variants of CYP2C9 are CYP2C9*1, CYP2C9*2, and CYP2C9*3. Some studies have shown that the metabolism of warfarin is reduced in patients with the CYP2C ${ }^{*} 3$ allele and that these patients therefore require a lower dose of the drug $(2,3)$. Compared to CYP2C9*1, CYP2C9*2 reduces

\footnotetext{
Address for correspondence: Dr. Demet Altun, Etimesgut Askeri Hastanesi, Pediyatrik Bölümü, Etimesgut, 06790 Ankara-Türkiye

Phone: +90 3122491011 - 3623 Fax: +90 3122444977 E-mail: draltundemet@gmail.com Accepted Date: 09.11.2015 Available Online Date: 
the metabolism of warfarin by $30 \%$, whereas CYP2C9*3 reduces it by $80 \%$. Thus, warfarin is more slowly metabolized in patients with these variants than in those without the variants. In addition, patients with these variants have lower dose requirements and are more vulnerable to the anticoagulation effects of the drug $(2,3,7,8)$.

Warfarin is a competitive inhibitor of VKOR, which is encoded by the VKORC1 gene. A missense mutation in codon 1639 of the VKORC1 gene is associated with decreased warfarin resistance, leading to different dose requirements $(1,2,9,10)$. The two variations of the VKORC1 enzyme, other than wild-type VKORC1 (GG), are VKORC1 (GA) and VKORC1 (AA). Latest studies have shown that patients with the VKORC1 (AA) polymorphism are most sensitive to warfarin and have the lowest dose requirements and that patients with the VKORC1 (GA) polymorphism are less sensitive to warfarin than normal and require average doses of warfarin $(1,2,9,10)$.

To our knowledge, few studies have been published on the frequency of the CYP2C9 and VKORC1 alleles and their effect on warfarin dose requirements in a pediatric population (11-13). Therefore, in this study, we evaluated the frequency and effect of the CYP2C9 and VKORC1 genetic polymorphisms in pediatric patients. This study aimed to determine the optimum dose of warfarin at the beginning of therapy, thereby reducing the rate of complications and enhancing the efficacy of the treatment.

\section{Methods}

\section{Study design}

This study was a prospective observational study. It was conducted after obtaining approval from the Institutional Review Board of Gazi University Faculty of Medicine Ethical Committee. Written informed consent was obtained from all participants and their families after a full discussion of the aim of the study.

\section{Study population}

This was a multicenter study. Fifty-eight blood samples (from 31 males and 27 females) were collected from patients attending the Departments of Pediatric Cardiology of Gazi University, Yüksek Ihtisas Education and Research Hospital, Başkent University Hospital, Dışkapı Children's Hospital, and Sami Ulus Children's Training and Research Hospital. As a control group, blood samples were collected from 149 healthy participants (63 males and 86 females) undergoing follow-up at Gazi University Department of Pediatrics in Turkey. The age range of the patients was $0.2-18$ years. The indications for warfarin use in the patients were cardiac valvular disease in $44.82 \%(n=26)$ of the patients, thrombophilia in $18.96 \%$ ( $n=11)$, aortic valve replacement in $13.79 \%(n=8)$, dilated cardiomyopathy in $8.62 \%(n=5)$, mitral valve replacement in $6.89 \%(n=4)$, and other conditions in $6.89 \%(n=4)$. Patients with INRs between 2 and 3 were included.

All patients underwent standard biochemical tests, and data on their age, height, weight, body surface area, comorbidity, concomitant medications, INRs, indication for warfarin use, starting date of the medication, and screened polymorphisms were recorded.
The patients were advised to avoid specific dietary supplements, including vitamin $\mathrm{K}$, which reduces the effect of warfarin.

Patients who were receiving drugs that interact with warfarin (CYP2C9 metabolized drugs), who smoke, and who had chronic liver or renal disease, obesity, hypothyroidism, or hyperthyroidism by either obtaining this information from the history of the patient or on physical examination were excluded.

\section{Genotyping}

Five milliliters of blood were drawn from each patient and the healthy controls and collected in sodium citrate tubes. The blood samples were centrifuged at $3000 \mathrm{x} \mathrm{g}$ for $10 \mathrm{~min}$ to separate the plasma. Genomic DNA was isolated from peripheral lymphocytes using a Macherey-Nagel genomic DNA purification kit (Macherey-Nagel GmbH \& Co. KG, Germany).

The polymerase chain reaction (PCR)-restriction fragment length polymorphism (RFLP) method was used for the determination of genetic polymorphisms in VKORC1 and CYP2C9 (the wild-type CYP2C9*1 allele and CYP2C9*2 and CYP2C9*3 variants) (14). Real time-PCR (RT-PCR) was used to confirm (14).

\section{Estimating the ideal warfarin dose}

A multivariate regression model developed by Sconce et al. (2) in 2005 was used to estimate the warfarin doses, as it has the largest $\mathrm{R} 2$ value. The model includes the following variables: age, CYP2C9 and VKORC1 genotypes, and height.

$\sqrt{\text { Dose }}=0.628-0.0135$ (age) -0.240 (CYP2C9*2) -0.370

(CYP2C9*3) -0.241 (VKORC1) +0.0162 (height)

In the equation shown above, age was calculated in years, and height was calculated in centimeters. For the CYP2C9*2 and CYP2C9*3 genotypes, 0 (a homozygote normal allele $=$ wild-type), 1 (a heterozygote allele), and 2 (a homozygote mutant allele) were the inputs. For the VKORC1 genotype, 1 (GG allele; wild-type), 2 (GA allele; heterozygote), and 3 (AA allele; homozygote) were the inputs (2).

\section{Estimating the mean administered warfarin dose}

Both the daily prescribed warfarin doses and INRs of the patients were recorded. For patients with INRs between 2 and 3 for at least one month, the warfarin doses were recorded, and the average dose was obtained. The mean administered warfarin dose (Mean \pm SD) of all patients was $4.21 \pm 1.29 \mathrm{mg} /$ daily. The average administered doses were compared with those of the ideal warfarin doses calculated with the equation. When comparing the average values with those of the ideal warfarin doses, 11 patients were not included in the analysis because of inadequate data.

\section{Statistical analysis}

Statistical analysis was carried out with SPSS (Statistical Package for Social Sciences) for Windows 11.5. Whether the differences between mean daily ideal dose and mean daily administered dose were statistically significant or not was evaluated 
by paired samples t-tests. Nominal data were evaluated using logistic regression analysis. All single nucleotide polymorphisms were tested for deviations from the Hardy-Weinberg disequilibrium. While the mean difference in administered doses between wild-types and heterozygotes was compared by student's t test, otherwise, one-way analysis of variance (ANOVA) was used to compare the administered doses of the wild-type, heterozygote, and mutant groups. Degrees of association between continuous variables were evaluated by Pearson's correlation analysis. A pvalue $>0.05$ was considered as statistically significant.

\section{Results}

The study consisted of 58 patients, 31 males $(53.4 \%)$ and 27 females $(46.6 \%)$, and 149 healthy controls, 63 males $(42.3 \%)$ and 86 females $(57.7 \%)$. The age range of the patients was $0.2-18$ years, with a mean age of $13.4 \pm 4.7$ years. The age range of the healthy controls was $0.5-18$ years, with a mean age of $9.7 \pm 4.8$ years. The weight and height percentiles of the patients were between the $10^{\text {th }}$ and $75^{\text {th }}$ percentiles. The mean $B M I$ was $19.33 \pm 3.76 \mathrm{~kg} / \mathrm{m}^{2}$ (men) and $18.42 \pm 5.42 \mathrm{~kg} / \mathrm{m}^{2}$ (women). The effects of the person's age, weight, and body surface area on warfarin dose management were evaluated. The mean daily administered warfarin dose was also increased with increasing age, weight, and BMI $(r=0.585, p<0.001 ; r=0.61, p<0.001$ and $r=0.427$, $p=0.003$, respectively).

The genotype distribution of CYP2C9*2, CYP2C9*3, and VKORC1 in our pediatric study population was calculated. None of the patients or controls had a homozygous mutation in the CYP2C9*2 gene. One hundred thirteen $(54.6 \%)$ had the CYP2C9 ${ }^{*} 1 /{ }^{*} 1$ allele, $50(24.2 \%)$ had the $* 1 /{ }^{*} 3$ allele, $4(1.9 \%)$ had the $* 3 / * 3$ allele, $34(16.4 \%)$ had the ${ }^{*} 1 /{ }^{*} 2$ allele, and $6(2.9 \%)$ had the $* 2 / * 3$ allele. Regarding the VKORC1 gene polymorphism, $55(26.6 \%)$ had the wild-type allele, $109(52.7 \%)$ had the heterozygous allele, and $43(20.8 \%)$ had the mutant allele (Table 1$)$.

The comparison of the mean administered warfarin doses with those of the estimated ideal doses was shown in Table 2. The mean daily ideal warfarin dose in the cases with the wild-type CYP2C9*2 allele was significantly higher than that of the mean daily administered dose $(p<0.001)$. However, there was no statistically significant difference between the mean daily ideal dose and the mean daily administered dose in patients with the CYP2C9*2 heterozygote allele. In contrast, the mean daily ideal warfarin dose in patients with the wild-type CYP2C9*3 allele and heterozygote allele was significantly higher than that of the mean daily administered dose $(p<0.001)$. The mean daily ideal warfarin dose in patients with the wild-type VKORC1 allele and heterozygote allele was significantly higher than that of the mean daily administered dose. Conversely, there was no statistically significant difference between the doses in patients with the VKORC1 mutant allele.

The correlation coefficient revealed a correlation between the mean ideal dose and the administered dose in all the patients (Table 3); this statistic was found to be 0.64 . The results of
Table 1. Distribution of CYP2C9*2, CYP2C9*3 and VKORC1 genotypes in our pediatric study population

\begin{tabular}{|l|c|c|}
\hline \multirow{3}{*}{ Gene } & Genotype & Frequency, $\mathbf{n}(\%)$ \\
\cline { 2 - 3 } & & Population (n=207) \\
\hline \multirow{3}{*}{ CYP2C9 } & ${ }^{*} 1 /{ }^{*} 1$ & $113(54.6 \%)$ \\
\cline { 2 - 3 } & ${ }^{*} 1 /{ }^{*} 3$ & $50(24.2 \%)$ \\
\cline { 2 - 3 } & ${ }^{*} 3 /{ }^{*} 3$ & $4(1.9 \%)$ \\
\cline { 2 - 3 } & ${ }^{*} 1 /{ }^{*} 2$ & $34(16.4 \%)$ \\
\hline \multirow{3}{*}{ VKORC1 } & ${ }^{*} 2 /{ }^{*} 3$ & $6(2.9 \%)$ \\
\cline { 2 - 3 } & Wild-type & $55(26.6 \%)$ \\
\cline { 2 - 3 } & Heterozygote & $109(52.7 \%)$ \\
\cline { 2 - 3 } CYP2C9 - cytochrome P450 2C9; VKORC1 - vitamin K epoxide reductase complex subunit 1 \\
\cline { 2 - 3 }
\end{tabular}

Table 2. Comparison of mean administered and ideal warfarin doses in patients carrying different genotypes

\begin{tabular}{|c|c|c|c|c|}
\hline \multirow[t]{2}{*}{ Gene } & \multirow[t]{2}{*}{ Genotype } & \multicolumn{2}{|c|}{$\begin{array}{l}\text { Warfarine dose, mg/daily } \\
\text { (Mean } \pm \text { SD) }\end{array}$} & \multirow[t]{2}{*}{$P^{\dagger}$} \\
\hline & & $\begin{array}{l}\text { Administered } \\
\text { dose }\end{array}$ & $\begin{array}{l}\text { Ideal } \\
\text { dose }\end{array}$ & \\
\hline \multirow{2}{*}{ CYP2C9*2 } & Wild-type & $4.35 \pm 1.25$ & $5.18 \pm 1.63$ & $<0.001^{\#}$ \\
\hline & Heterozygote & $3.67 \pm 1.38$ & $4.06 \pm 1.74$ & 0.193 \\
\hline \multirow{2}{*}{ СYP2C9*3 } & Wild-type & $4.40 \pm 1.39$ & $5.22 \pm 1.73$ & $<0.001^{\#}$ \\
\hline & Heterozygote & $3.98 \pm 1.13$ & $4.76 \pm 1.60$ & $0.003^{\#}$ \\
\hline \multirow{3}{*}{ VKORC1 } & Wild-type & $4.80 \pm 1.55$ & $5.81 \pm 1.99$ & $0.035^{\#}$ \\
\hline & Heterozygote & $4.26 \pm 1.24$ & $5.00 \pm 1.58$ & $<0.001^{\#}$ \\
\hline & Mutant & $3.58 \pm 1.09$ & $4.05 \pm 1.54$ & 0.328 \\
\hline All patients & & $4.21 \pm 1.29$ & $4.94 \pm 1.70$ & $<0.001^{\#}$ \\
\hline \multicolumn{5}{|c|}{$\begin{array}{l}\text { \#: P<0.05; † paired samples t-test. CYP2C9*2 - cytochrome P450 2C9*2 allele; } \\
\text { CYP2C9*3 - cytochrome P450 2C9*2 allele; VKORC1 - vitamin K epoxide reductase } \\
\text { complex subunit } 1\end{array}$} \\
\hline
\end{tabular}

Table 3. Correlation between administered and ideal warfarin doses in patients carrying different genotypes

\begin{tabular}{|l|c|c|c|c|}
\hline Gene & Genotype & $\begin{array}{c}\text { Intraclass } \\
\text { correlation }\end{array}$ & $\begin{array}{c}\text { 95\% confidence } \\
\text { intervals }\end{array}$ & $\boldsymbol{P}^{\dagger}$ \\
\hline \multirow{2}{*}{ CYP2C9*2 } & Wild-type & 0.570 & $0.308-0.752$ & $<0.001^{\#}$ \\
\cline { 2 - 5 } & Heterozygote & 0.838 & $0.500-0.956$ & $<0.001^{\#}$ \\
\hline \multirow{2}{*}{ CYP2C9*3 } & Wild-type & 0.692 & $0.427-0.848$ & $<0.001^{\#}$ \\
\cline { 2 - 5 } & Heterozygote & 0.626 & $0.262-0.836$ & $<0.001^{\#}$ \\
\hline \multirow{3}{*}{ VKORC1 } & Wild-type & 0.699 & $0.110-0.930$ & $0.013^{\#}$ \\
\cline { 2 - 5 } & Heterozygote & 0.654 & $0.388-0.821$ & $<0.001 \#$ \\
\cline { 2 - 5 } & Mutant & 0.407 & $<0-0.808$ & 0.097 \\
\hline All patients & & 0.645 & $0.444-0.785$ & $<0.001^{\#}$ \\
\hline \multirow{2}{*}{ P P<0.05;: } & & &
\end{tabular}

\#: $\mathrm{P}<0.05 ;{ }^{\dagger}$ : Intraclass correlation analysis. CYP2C9*2 - cytochrome $\mathrm{P} 4502 \mathrm{C} 9 * 2$ allele; CYP2C9*3 - cytochrome P450 2C9 *2 allele; VKORC1 - vitamin K epoxide reductase complex subunit 1 
the ANOVA of the administered doses in the wild-type, heterozygote, and mutant groups of genetic polymorphisms revealed no statistically significant differences.

The comparison of the results obtained using PCR-RFLP with those obtained by RT-PCR also showed no significant difference. A minimum of 2 days is needed to obtain the results of PCR-RFLP, whereas the results of RT-PCR were obtained within as little time as $1.5 \mathrm{~h}$. Thus, the reliability of RT-PCR was confirmed; this method is faster.

\section{Discussion}

CYP2C9 and VKORC1 polymorphisms vary according to ethnicity (15), and they can be used as a dose-adjustment factor in patients receiving warfarin. We examined the prevalence and diversity of genetic polymorphisms in our pediatric population and compared these with findings reported in other studies $(9,16,17)$.

In the present study, CYP2C9*2 and CYP2C9*3 gene regions were dealt with together in the entire study population in order to facilitate comparisons with previous studies. Aynacıoğlu et al. (16) examined the frequencies of the CYP2C9*1, CYP2C9*2, and CYP2C $9 * 3$ alleles and demonstrated for their adult patients similar results to those of our study using the PCR-RFLP method in a population from the Southeastern Anatolian region of Turkey in 1999. They reported that the frequency of the wild $1 *$ allele $\left({ }^{*} 1 /{ }^{*} 1\right)$ was $61.72 \%$. In their study, the frequency of genotypes heterozygous for the 2 allele $\left({ }^{*} 1 /{ }^{*} 2\right)$ was $18.04 \%$. The frequency of genotypes heterozygous for the ${ }^{*} 3$ allele $\left(* 1 /{ }^{*} 3\right)$ was $17.23 \%$ and homozygous for the ${ }^{*} 2$ allele $\left({ }^{*} 2 /{ }^{*} 2\right)$ and ${ }^{*} 3$ allele $\left({ }^{*} 3 /{ }^{*} 3\right)$ was $1 \%$ and $0.8 \%$, respectively, accounting for $38.28 \%$ of the variation in total (16). When we compare our results with those of Aynacıoğlu et al. (16), the incidence of the ${ }^{*} 1 /{ }^{*} 3$ and ${ }^{*} 2 / * 3$ alleles was higher in our study, and we did not detect any ${ }^{*} 2 /{ }^{*} 2$ alleles.

We compared the frequencies of CYP2C9 polymorphisms in our population with those of several Asian populations (Korean, Japanese, Malaysian) described in other studies (18-21). The average frequency of the wild allele $* 1 /{ }^{*} 1$ was $90 \%$ in the Asian population. The incidence of this polymorphism in our population was substantially higher.

Regarding the VKORC1 gene polymorphism, in an another study of a Turkish adult population, Öner Özgön et al. (22) reported that the frequency of the wild VKORC1-1639 G/G allele was $28.8 \%$, whereas that of the heterozygous $\mathrm{G} / \mathrm{A}$ allele and homozygous mutant allele, $\mathrm{A} / \mathrm{A}$, was $42.4 \%$ and $28.8 \%$, respectively. These results are similar to the frequencies of the VKORC1 genotype that we found in our pediatric study.

Nowak-Gottl et al. (9) investigated the frequency of VKORC1 polymorphisms in a group of Caucasian pediatric patients. Compared to their findings, the incidence of the wild VKORC1-1639 $\mathrm{G} / \mathrm{G}$ allele in our pediatric population was lower due to a higher incidence of the homozygous mutant allele, $1639 \mathrm{~A} / \mathrm{A}$. The incidence of CYP2C $9 * 3$ was also higher in our population.
Nahar et al. (17) conducted a pilot study of the predicted warfarin response of healthy South and North Indians based on the presence/absence of polymorphisms. South and North Indians are two ethno-geographically different populations and the frequency of the CYP2C9 ${ }^{*} 2$ allele and ${ }^{*} 1 /{ }^{*} 1, * 1 /{ }^{*} 2$, and ${ }^{*} 3 /{ }^{*} 3$ genotypes were found to be different in these populations. The frequency of the CYP2C9*3 allele was higher than that in other Asian populations, but (10\%) was similar to that in Caucasians. The frequency of the VKORC1-1639A allele (17\%) was observed to be much lower than that in the Asian populations, Caucasians, and our study population (17).

As a result, CYP2C9 and VKORC1 genetic polymorphisms in Turkish society show the most similarities with Caucasians among the ethnic groups $(9,17)$.

According to the Food and Drug Association (FDA), warfarin is one of the top 10 most prescribed drugs, and it has the most side effects. The FDA recommends that clinicians should consider genetic testing of CYP2C9 and VKORC1 polymorphisms in every patient before adjusting the warfarin dose $(20,23)$. Although there are many studies in the literature on the impact of genetic polymorphisms on warfarin dose requirements in adult populations $(14,16,20,22,24,25)$, there are only a few studies of pediatric populations (11-13). In children, data about the pharmacogenetics of warfarin are limited and inconsistent.

Sconce et al. (2) studied the effect of the CYP2C9 and VKORC1 $(-1639)$ genetic polymorphisms on warfarin dose requirements in adults. They showed that patients with a mutation in both genes required a lower warfarin dose. They determined that a patient's age, height, and CYP2C9 and VKORC1 gene mutations are the most important factors in the maintenance of warfarin doses and formulated a way to calculate the ideal warfarin dose (2). We used this formula for estimating the ideal warfarin doses for the patients in our study. We found a significant correlation between the mean ideal dose and the administered dose in all patients. Clinicians should keep in mind the importance of these genetic polymorphisms before commencing warfarin treatment. The correlation coefficient showed concordance between the mean ideal dose and the administered dose in all patients. Consequently, sufficient accommodation was observed between the doses.

To our knowledge, our study is the first of its kind in a pediatric population in Turkey. We evaluated the effect of an individual's age, weight, and BMI on warfarin dose management in addition to the effect of their polymorphisms. Our study showed that the mean daily dose of warfarin was higher in patients with the CYP2C9 wild-type genotype than in those with the variant *2 and *3 alleles. The average daily warfarin dose required for maintenance therapy in patients with the CYP2C9*2 polymorphism mutation was $3.67 \pm 1.38$, whereas it was $4.35 \pm 1.25$ in those without this mutation. The average dose in patients with the CYP2C9*3 mutation was $3.98 \pm 1.1 .3$, whereas it was $4.40 \pm 1.39$ in those without this mutation. However, this difference was not statistically significant.

In a study on Japanese pediatric patients in 2010, Kato et al. (12) found that the VKORC1 genetic polymorphism and age are 
the two main factors that affect warfarin dose requirements. In the present study, we detected that weight and BMI, in addition to age, were affected the mean daily administered warfarin dose. In a preliminary study on 41 Egyptian pediatric patients receiving warfarin maintenance therapy, Kamel El-Din et al. (11) found that age is the most significant determinant of the warfarin dose and that the presence of the CYP2C9 and VKORC1 gene polymorphisms does not affect the warfarin dose. In contrast, in a recent study on Egyptian patients, Azzam et al. (24) suggested that the VKORC1-1639 GG and wild-type CYP2C9*1*1 genotypes are associated with high dose requirements during warfarin therapy and that VKORC1-1639 GG is responsible for warfarin resistance and failure. We considered CYP2C9 and VKORC1 genetic polymorphisms in addition to age and height, while the ideal dose requirements were calculated.

Warfarin dose requirements for patients carrying both CYP2C9 and VKORC1 polymorphisms were lower than those for patients with the wild allele, and those patients have a greater tendency to experience hemorrhagic complications during warfarin initiation (26), in agreement with the findings by Sconce et al. (2). The authors concluded that the VKORC1 polymorphism plays a bigger role than the CYP2C9 polymorphism in warfarin dose variability, in agreement with the findings by Özer et al. (14). In a study conducted in 2007, Muzskat et al. (3) reported that patients with the CYP2C9*3 allele required $33 \%$ less warfarin than those with the wild type. Sanderson et al. (27) found that warfarin dose requirements decreased $17 \%$ in the presence of the CYP2C9*3 polymorphism and $37 \%$ in the presence of the CYP2C9*2 polymorphism. In another recent study, Shaw et al. (13) confirmed the importance of the VKORC1/CYP2C9 genotypes in warfarin dosing in a young pediatric cohort and demonstrated the impact of genetic polymorphisms on clinical outcomes in their study group. In the present study, considering the dose given to the patient, we found that patients harboring the genetic polymorphisms received lower doses of warfarin than those with the wild allele, a result consistent with those of the abovementioned authors.

\section{Study limitations}

The main limitation was the relatively small sample size. Further randomized studies involving a larger number of children are warranted to determine the true impact of genetic factors on warfarin doses in Turkish pediatric patients.

\section{Conclusion}

The incidences of CYP2C9 and VKORC1 polymorphisms in our study population were higher than those found in many other populations. As a higher incidence of these polymorphisms increases the risk of bleeding complications, by detecting these polymorphisms in patients before warfarin treatment, clinicians can adjust the warfarin dose to the individual and prevent such complications. In the present study, there was a relatively high correlation (64.5\%) between the ideal dose and the administered dose. Given this finding, clinicians should take into account CPY2C9 and VKORC1 genetic polymorphisms when considering the ideal dose of warfarin for each patient.

Conflict of interest: None declared.

Peer-review: Externally peer-reviewed.

Authorship contributions: Concept- S.K., M.A.E., R.O., F.S.T., A.D.O., T.G.; Design- B.D.T., S.K., M.A.E., R.O., F.S.T., A.D.O., T.G.; Supervision- S.K., M.A.E., B.D.T., R.O., E.S.T., A.D.O., T.G.; Materials- S.K., B.D.T., M.A.E., R.O., T.G., A.D.O., F.S.T.; Data collection \&/or processing - S.K., B.D.T., M.A.E., R.O., T.G., A.D.O., F.S.T., D.A.; Analysis and/or interpretation- B.D.T., S.K., M.A.E, D.A.; Literature search- B.D.T., S.K., M.A.E., D.A.; Writing - B.D.T., S.K., M.A.E.; Critical review- B.D.T., S.K., M.A.E., R.O., F.S.T., A.D.O.

\section{References}

1. Oldenburg J, Watzka M, Rost S, Muller CR. VKORC1: Molecular target of coumarins. J Thromb Haemost 2007; 5: 1-6.

2. Sconce EA, Khan TI, Wynne HA, Avery P, Monkhouse L, King BP, et al. The impact of CYP2C9 and VKORC1 genetic polymorphism and patient characteristics upon warfarin dose requirements: proposal for a new dosing regimen. Blood 2005; 106: 2329-33.

3. Muszkat M, Blotnik S, Elami A, Krasilnikov I, Caraco Y. Warfarin metabolism and anticoagulant effect: a prospective, observational study of the impact of CYP2C9 genetic polymorphism in the presence of drug-disease and drug-drug interactions. Clin Ther 2007; 29: 427-37.

4. Hirsh J, Dalen J, Anderson DR, Poller L, Bussey H, Ansell J, et al. Oral anticoagulants: mechanism of action, clinical effectiveness, and optimal therapeutic range. Chest 2001; 119: 8-21.

5. Streif W, Andrew M, Marzinotto V, Massicotte P, Chan AK, Julian $\mathrm{JA}$, et al. Analysis of warfarin therapy in pediatric patients: A prospective cohort study of 319 patients. Blood 1999; 94: 3007-14.

6. Gage BF, Lesko LJ. Pharmacogenetics of warfarin: regulatory, scientific, and clinical issues. J Thromb Thrombolysis 2008; 25: 45-51.

7. Takanashi K, Tainaka H, Kobayashi K, Yasumori T, Hosakawa M, Chiba K. CYP2C9 Ile359 and Leu359 variants: enzyme kinetic study with seven substrates. Pharmacogenetics 2000; 10: 95-104.

8. Margaglione M, Colaizzo D, D'Andrea G, Brancaccio V, Ciampa A, Grandone $\mathrm{E}$, et al. Genetic modulation of oral anticoagulation with warfarin. Thromb Haemost 2000; 84: 775-8.

9. Nowak-Gottl U, Dietrich K, Schaffranek D, Eldin NS, Yasui Y, Geisen

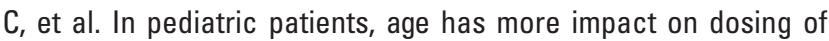
vitamin $\mathrm{K}$ antagonists than VKORC1 or CYP2C9 genotypes. Blood 2010; 116: 6101-5.

10. Wiwanitkit V. Pharmacogenomic effect of cytochrome P450 2C9 polymorphisms in different populations. Clin Appl Thromb Hemost 2006; 12: 219-22.

11. Kamal El-Din MA, Farhan MS, El Shiha RI, El-Kaffas RM, Mousa SM. Frequency of CYP2C9 and VKORC1 gene polymorphisms and their influence on warfarin dose in Egyptian pediatric patients. Paediatr Drugs 2014; 16: 337-41.

12. Kato Y, Ichida F, Saito K, Watanabe K, Hirono K, Miyawaki T, et al. Effect of the VKORC1 genotype on warfarin dose requirements in Japanese pediatric patients. Drug Metab Pharmacokinet 2011; 26: 295-9. 
13. Shaw K, Amstutz U, Hildebrand C, Rassekh SR, Hosking M, Neville $\mathrm{K}$, et al. VKORC1 and CYP2C9 genotypes are predictors of warfarinrelated outcomes in children. Pediatr Blood Cancer 2014; 61: 105562.

14. Özer N, Cam N, Tangürek B, Özer S, Uyarel H, Öz D, et al. The impact of CYP2C9 and VKORC1 genetic polymorphism and patient characteristics upon warfarin dose requirements in an adult Turkish population. Heart Vessels 2010; 25: 155-62.

15. Ansell J, Hirsh J, Dalen J, Bussey H, Anderson D, Poller L, et al. Managing oral anticoagulant therapy. Chest 2001; 119: 22-38.

16. Aynacıoğlu AS, Brockmoller J, Bauer S, Sachse C, Güzelbey P, Öngen Z, et al. Frequency of cytochrome P450 CYP2C9 variants in a Turkish population and functional relevance for phenytoin. $\mathrm{Br} \mathrm{J}$ Clin Pharmacol 1999; 48: 409-15.

17. Nahar R, Deb R, Saxena R, Puri RD, Verma IC. Variability in CYP2C9 allele frequency: a pilot study of its predicted impact on warfarin response among healthy South and North Indians. Pharmacol Rep 2013; 65: 187-94.

18. Yoon YR, Shon JH, Kim MK, Lim YC, Lee HR, Park JY, et al. Frequency of cytochrome P450 2C9 mutant alleles in a Korean population. Br J Clin Pharmacol 2001; 51: 277-80.

19. Gan GG, Phipps ME, Lee MM, Lu LS, Subramaniam RY, Bee PC, et al. Contribution of VKORC1 and CYP2C9 polymorphisms in the interethnic variability of warfarin dose in Malaysian populations. Ann Hematol 2011; 90: 635-41.

20. Miyagata Y, Nakai K, Sugiyama Y. Clinical significance of combined
CYP2C9 and VKORC1 genotypes in Japanese patients requiring warfarin. Int Heart J 2011; 52: 44-9.

21. Nasu K, Kubota T, Ishizaki T. Genetic analysis of CYP2C9 polymorphism in a Japanese population. Pharmacogenetics 1997; 7 : 405-9.

22. Öner Özgon $G$, Langaee $T Y$, Feng $H$, Buyru $N$, Ulutin T, Hatemi AC, et al. VKORC1 and CYP2C9 polymorphisms are associated with warfarin dose requirements in Turkish patients. Eur J Clin Pharmacol 2008; 64: 889-94.

23. Wysowski DK, Nourjah P, Swartz L. Bleeding complications with warfarin use: a prevalent adverse effect resulting in regulatory action. Arch Intern Med 2007; 167: 1414-9.

24. Azzam H, Elwakeel H, Awad I, El-Farahaty R, El-Gilany AH, ElSharawy S. VKORC1 and CYP2C9 genotypes in Egyptian patients with warfarin resistance. Blood Coagul Fibrinolysis 2014 Jun 27. Epub ahead of print.

25. Zhong SL, Liu Y, Yu XY, Xu D, Tan HH, Lin QX, et al. The influence of genetic polymorphisms and interacting drugs on initial response to warfarin in Chinese patients with heart valve replacement. Eur $\mathrm{J}$ Clin Pharmacol 2011; 67: 581-90.

26. Rieder MJ, Reiner AP, Gage BF, Nickerson DA, Eby CS, McLeod HL, et al. Effect of VKORC1 haplotypes on transcriptional regulation and warfarin dose. N Engl J Med 2005; 352: 2285-93.

27. Sanderson S, Emery J, Higgins J. CYP2C9 gene variants, drug dose, and bleeding risk in warfarin-treated patients: a HuGEnet systematic review and meta-analysis. Genet Med 2005; 7: 97-104. 